\title{
Correction to: The impact of low-volume metastasis on disease-free survival of women with early-stage cervical cancer
}

\author{
Alessandro Buda ${ }^{1}$ (1) $\cdot$ Jvan Casarin $^{2} \cdot$ Michael Mueller $^{3} \cdot$ Francesco Fanfani $^{4,5} \cdot$ Ignacio Zapardiel $^{6} \cdot$ Liliana Mereu $^{7}$. \\ Andrea Puppo ${ }^{8}$. Elena De Ponti ${ }^{9} \cdot$ Marco Adorni $^{1} \cdot$ Debora Ferrari $^{1} \cdot$ Maria Luisa Gasparri $^{10} \cdot$ Fabio Ghezzi $^{2}$. \\ Giovanni Scambia ${ }^{4,5} \cdot$ Andrea Papadia ${ }^{3,10}$
}

Published online: 21 November 2020

(c) Springer-Verlag GmbH Germany, part of Springer Nature 2020

\section{Correction to: \\ Journal of Cancer Research and Clinical Oncology https://doi.org/10.1007/s00432-020-03435-z}

In the original article, the name of the second author is incorrect.

The correct name is Jvan Casarin.

The original article has been corrected.

Publisher's Note Springer Nature remains neutral with regard to jurisdictional claims in published maps and institutional affiliations.

The original article can be found online at https://doi.org/10.1007/ s00432-020-03435-z.

Alessandro Buda

alebuda1972@gmail.com

1 Gynecology Oncology Surgical Unit, Department of Obstetrics and Gynecology, San Gerardo Hospital, University of Milano-Bicocca, Monza, Italy

2 Obstetrics and Gynecology Department, University of Insubria, Varese, Italy

3 Department of Obstetrics and Gynecology, University Hospital of Bern and University of Bern, Bern, Switzerland

4 Dipartimento della salute della donna, del bambino e di sanità pubblica, Fondazione Policlinico Universitario A. Gemelli IRCCS, Rome, Italy

5 Dipartimento Scienze della vita e sanità pubblica, Sacred Heart Catholic University, Rome, Italy
6 Gynecologic Oncology Unit, La Paz University Hospital-IdiPAZ, Madrid, Spain

7 Department of Obstetrics and Gynecology, Santa Chiara Hospital, Trento, Italy

8 Clinic of Obstetrics and Gynecology, Santa Croce e Carle Hospital, Cuneo, Italy

9 Department of Physical Medicine, San Gerardo Hospital, University of Milano-Bicocca, Monza, Italy

10 Department of Gynecology and Obstetrics, University of the Italian Switzerland (USI), Ente Ospedaliero Cantonale of Lugano, Lugano, Switzerland 